Revista de Metalurgia 52(4)

Octubre-Diciembre 2016, e083

ISSN-L: 0034-8570

doi: http://dx.doi.org/10.3989/revmetalm.083

\title{
Caracterización de escorias de cobre de fundiciones chilenas del Siglo XIX
}

\author{
Amin Nazer ${ }^{\mathrm{a}, \mathrm{b}}$, Jordi Payáa ${ }^{\mathrm{a}}$, María Victoria Borrachero ${ }^{\mathrm{a}}$, José Monzó ${ }^{\mathrm{a}, \bigotimes}$ \\ ${ }^{a}$ Universitat Politècnica de València, Instituto de Ciencia y Tecnología del Hormigón (ICITECH), Camino de Vera, s/n, \\ 46022 Valencia \\ ${ }^{\mathrm{b}}$ Universidad de Atacama, Facultad Tecnológica, Departamento de Construcción, Copayapu 485, \\ Copiapó, Región de Atacama, Chile \\ $\bowtie$ Autor para la correspondencia: jmmonzo@cst.upv.es
}

Enviado: 27 Mayo 2016; Aceptado: 6 Noviembre 2016; Publicado on-Line: 19 Diciembre 2016

RESUMEN: El objetivo de este trabajo es caracterizar 4 escorias de fundiciones de cobre del siglo XIX, procedentes de vertederos abandonados en la Región de Atacama - Chile, utilizando las técnicas de fluorescencia de rayos X (FRX), difracción de rayos X (DRX), microscopía electrónica de barrido (SEM), análisis de partículas por difracción láser (ADL), espectrometría infrarroja por transformadas de Fourier (FTIR) y análisis termogravimétrico (ATG). Las escorias de cobre estudiadas fueron clasificadas químicamente como escorias ácidas, con mayor contenido de $\mathrm{SiO}_{2}(38-49 \%)$ que de $\mathrm{Fe}_{2} \mathrm{O}_{3}(18-37 \%)$, y con una importante cantidad de $\mathrm{CaO}$ $(8-26 \%)$ y $\mathrm{Al}_{2} \mathrm{O}_{3}(5-8 \%)$. Su mineralogía y estructura es variada, presentando una de ellas una estructura amorfa y las tres restantes, una estructura cristalina con cierto carácter amorfo. Las fases minerales mayoritarias presentes en las escorias de cobre son diópsido, fayalita, magnetita, cristobalita y clinoferrosilita. Los niveles de calcio indicarían que las escorias poseen propiedades cementantes para ser utilizadas en materiales de construcción. Además, la importante cantidad de escoria disponible y el contenido de $\mathrm{CuO}(0,6-1,2 \%)$ muestran que puede ser de interés como materia prima en la recuperación del metal.

PALABRAS CLAVE: Caracterización; Escorias de cobre; Fundiciones chilenas; Vertederos abandonados

Citation / Cómo citar este artículo: Nazer, A., Payá, J., Borrachero, M.V., Monzó, J. (2016) "Caracterización de escorias de cobre de fundiciones chilenas del Siglo XIX”. Rev. Metal. 52(4):e083. doi: http://dx.doi.org/10.3989/revmetalm.083

\begin{abstract}
Characterization of chilean copper slag smelting nineteenth century. The aim of this work is to characterize four copper smelters slag nineteenth century, from abandoned landfills in Atacama Region - Chile, using the techniques of X-ray fluorescence (XRF), X-ray diffraction (XRD), scanning electron microscopy (SEM), particle analysis by laser diffraction (ADL), Fourier Transform Infrared Spectroscopy (FTIR) and thermogravimetric analysis (TGA). Copper slags studied were chemically classified as acidic slags, this slags presented higher $\mathrm{SiO}_{2}$ content (38-49\%) than $\mathrm{Fe}_{2} \mathrm{O}_{3}(18-37 \%)$ and a significant amount of $\mathrm{CaO}(8-26 \%)$ and $\mathrm{Al}_{2} \mathrm{O}_{3}$ $(8.5 \%)$. Mineralogy and structure was varied, presenting one of them an amorphous structure and the remaining three, a crystalline structure with partially amorphous character. The majority mineral phases presented in the copper slag were diopside, fayalite, magnetite, cristobalite and clinoferrosilita. Calcium levels indicate that the slags could have cementitious properties for use as a binder in construction materials. Moreover, the significant amount of slag available and $\mathrm{CuO}$ content $(0.6-1.2 \%)$ show that may be of interest as raw material for metal recovery.
\end{abstract}

KEYWORDS: Abandoned landfills; Characterization; Chilean smelters; Copper slag

Copyright: (C) 2016 CSIC. Este es un artículo de acceso abierto distribuido bajo los términos de la licencia Creative Commons Attribution (CC BY) España 3.0. 


\section{INTRODUCCIÓN}

La escoria de cobre (EC), es un residuo industrial perteneciente al grupo de las escorias no ferrosas y es generada durante el proceso de producción de ánodos de cobre. Las fundiciones primarias modernas generan entre 2,2 y 3 ton de EC por cada tonelada de cobre producida (Shi et al., 2008), mientras que una fundición de comienzos del siglo XX (Ambler, 1920), nos permite estimar que esta relación fue 2,5 .

Chile es un país con tradición minera-metalúrgica, actividad productiva que ha tenido un desarrollo importante desde mediados del siglo XIX hasta nuestros días. Antecedentes bibliográficos describen que en el Norte de Chile, particularmente en las regiones de Coquimbo y Atacama, se establecieron numerosas fundiciones cercanas a los yacimientos mineros de cobre, llegándose a registrar en el año 1858, 145 fundiciones de cobre con un total de 347 hornos (Sociedad Nacional de Minería, 1894). Estas fundiciones generaron EC, las cuales han permanecido en los vertederos como depósitos abandonados desde entonces, causando efectos en el paisaje, en el suelo y agua, económicos e impacto social.

La valorización de estos residuos puede traer beneficios económicos y medioambientales. Las EC modernas se comercializan en los sectores minería y construcción. En la construcción se utiliza principalmente como material de relleno y como material abrasivo (Murari et al., 2015). En la literatura científica se reportan pocos estudios sobre caracterización de EC antiguas (Ros-Latienda y FernándezCarrasquilla, 2013) y del uso de EC de vertederos antiguos como un material conglomerante (Nazer et al., 2016). Otros estudios determinaron las propiedades cementantes de las EC en la fabricación de morteros (Ariño y Mobasher, 1999; Tixier et al., 1997; Taha et al., 2007; Sánchez de Rojas et al., 2008; Nazer et al., 2013) y hormigones (Al-Jabri et al., 2011; Thomas and Gupta, 2013; Mithun and Narasimhan, 2016).

Por otra parte, la posibilidad de valorizar las EC mediante la recuperación de metales contenidos en ellas, puede ser una interesante alternativa para la industria metalúrgica. Diversos estudios proponen metodologías para la recuperación de $\mathrm{Cu}$, Co y $\mathrm{Zn}$ (Arslan y Arslan, 2002; Banza et al., 2002; Yang et al., 2010b), Cu y Co (Rudnik et al., 2009), Si, Fe y $\mathrm{Cu}$ (Chen et al., 2011), $\mathrm{Cu}$ y Fe (Cao et al., 2012), $\mathrm{Fe}$ (Kim et al., 2013; Li et al., 2013), Cu, Zn y Fe (Nadirov et al., 2013) y Cu (Panda et al., 2015), entre otros.

La composición química de las EC varía con el tipo de horno usado (Shi y Qian, 2000), el proceso metalúrgico y la composición del concentrado (Jun-wei, 2013). Para tener una aproximación de los tipos de hornos de una fundición chilena en el siglo XIX, la literatura nos muestra 4 tipos, pudiéndose usar uno o varios de ellos conjuntamente. El más antiguo fue el horno de manga o de viento (Marín Vicuña, 1920), luego hornos de reverbero en el cual cada horno contaba con su chimenea propia, y otro tipo de horno de reverbero en el que varios de estos hornos eran servidos por una sola chimenea. Además se contaba con el horno de calcinación que permitía eliminar los elementos volátiles de los concentrados de minerales como los sulfuros. La fundición del cobre en sí, practicaba normalmente tres fundiciones sucesivas de un mineral de partida conteniendo hasta un $13 \%$ de cobre. Con la primera fundición se obtenía el eje o régulo con $43-45 \%$ de cobre, material que pasaba a una segunda fusión para tener leyes que fluctuaban entre $68-70 \%$ de cobre. Con la tercera y última fundición se conseguían barras con una pureza de entre el 98-99\%. Posteriormente se innovó radicalmente reduciendo tan sólo a dos las fundiciones sucesivas del mineral, lo que trajo consigo un ahorro de tiempo en la producción de barras de cobre (Aracena, 1884). Estos procedimientos metalúrgicos del cobre usados en la segunda mitad del siglo XIX en Chile, generaban EC que contenían entre 0,4 y $0,6 \%$ de cobre, siendo la leña o el carbón mineral los combustibles que alimentaban los hornos de fundición (Aracena, 1884; Vicuña Mackenna, 1882).

Las EC pueden ser vertidas y enfriadas lentamente al aire, o pasar por un proceso de enfriamiento rápido con chorro de aire o agua. Las EC enfriadas al aire son de color negro vítreo mostrando baja absorción de agua, mientras que las que son enfriadas con agua son amorfas, granuladas y con mayor capacidad de absorción (Onuaguluchi, 2012). Es frecuente encontrar en las EC modernas altos contenidos de $\mathrm{SiO}_{2}(10-71 \%)$ y $\mathrm{FeO}(0,7-62 \%)$ y los elementos químicos predominantes corresponden al $\mathrm{Cu}, \mathrm{Pb}, \mathrm{Ni}$ y $\mathrm{Zn}$. Sus fases mineralógicas forman principalmente fayalita $\mathrm{y}$ magnetita (Piatak et al., 2015). Además algunas EC muestran un elevado contenido de $\mathrm{CaO}$, compuesto que otorgaría efectos positivos en las propiedades mecánicas de materiales conglomerantes para su uso como material de construcción (Choi y Lee, 2013; Khater, 2012). Al respecto, Nazer et al. (2016) mostraron que las EC poseen propiedades puzolánicas y la influencia del contenido de $\mathrm{CaO}$ de dos EC chilenas del siglo XIX contribuye en la resistencia mecánica de morteros de cemento Portland y de morteros con EC activadas alcalinamente (Nazer et al., 2016).

Con el propósito de aportar información que permita orientar los estudios sobre el potencial uso de las EC como material de construcción o como una materia prima en la extracción de metales secundarios para el sector metalúrgico, en este trabajo se caracterizan cuatro EC chilenas del 
siglo XIX procedentes de vertederos abandonados, mediante fluorescencia de rayos-X (FRX), difracción de rayos-X (DRX), espectroscopia infrarroja por transformada de Fourier (FTIR), análisis termogravimétrico (ATG), microscopía electrónica de barrido de emisión de campo (FESEM) y análisis de difracción láser (ADL).

\section{MATERIALES Y MÉTODOS}

\subsection{Materiales}

Se caracterizaron 4 EC de vertederos de fundiciones chilenas del siglo XIX las cuales son identificadas como Playa Negra (EC-PN), Púquios (EC-PQ), Canto del Agua (EC-CA) y Nantoco (EC-NT), nombres adoptados según la zona geográfica de su procedencia. En la Tabla 1 se muestran las coordenadas de ubicación de los escoriales estudiados. Se estima que la cantidad de EC del presente estudio, llegaría a aproximadamente a $\operatorname{los} 3,5 \times 10^{6}$ toneladas métricas (Nazer et al., 2012).

\subsection{Equipos y procedimientos}

\subsubsection{Toma de muestras in situ}

La toma de muestras de las EC antiguas desde los diferentes vertederos, se realizó mediante excavaciones hasta una profundidad media de $0,70 \mathrm{~m}$. Se descartaron las EC superficiales con el propósito de prescindir de muestras potencialmente meteorizadas o contaminadas con polvo u otras sustancias. Se practicaron 5 calicatas en el vertedero y desde cada una de ellas, se extrajo una cantidad de muestra que se depositó en bolsas plásticas totalizando $40 \mathrm{~kg}$ aproximadamente, las cuales fueron llevadas al laboratorio de Obras Civiles de la Universidad de Atacama, Chile, para homogeneizarlas. Posteriormente, la muestra de cada escoria fue mezclada, cuarteada y almacenada para su molienda.

\subsubsection{Molienda de la escoria}

En la Universidad de Atacama se trituraron las EC de manera secuencial en 2 diferentes machacadoras: de mandíbula y de rodillo, hasta alcanzar un tamaño medio de $35 \mu \mathrm{m}$. La muestra se homogeneizó en un cortador de Riffle (32" x 1/2")

TABla 1. Coordenadas de ubicación de escoriales de cobre

\begin{tabular}{lcc}
\hline Escorial & Latitud & Longitud \\
\hline EC-PN & $27^{\circ} 03^{\prime} 39^{\prime \prime} \mathrm{S}$ & $70^{\circ} 48^{\prime} 45^{\prime \prime} \mathrm{O}$ \\
EC-PQ & $27^{\circ} 07^{\prime} 00^{\prime \prime} \mathrm{S}$ & $69^{\circ} 53^{\prime} 56^{\prime \prime} \mathrm{O}$ \\
EC-CA & $28^{\circ} 09^{\prime} 04^{\prime \prime} \mathrm{S}$ & $70^{\circ} 54^{\prime} 37^{\prime \prime} \mathrm{O}$ \\
EC-NT & $27^{\circ} 32^{\prime} 30^{\prime \prime} \mathrm{S}$ & $40^{\circ} 16^{\prime} 11^{\prime \prime} \mathrm{O}$ \\
\hline
\end{tabular}

y alrededor de $2 \mathrm{~kg}$ de polvo de cada EC, se molieron posteriormente en los laboratorios de la Universitat Politècnica de València en España. La molienda fina se realizó en un molino de bolas Nannetti modelo Speedy 1. La escoria se depositó dentro de un recipiente de porcelana de un litro de capacidad, en el cual se introdujeron 60 bolas de alúmina de $18 \mathrm{~mm}$ de diámetro y $500 \mathrm{~g}$ de escoria, con un tiempo de molienda 35-55 min. Cada 5 min se extrajo una pequeña muestra del material molido verificándose su granulometría por el ensayo de análisis por difracción láser.

\subsubsection{Caracterización de las escorias de cobre}

En la determinación de la distribución de tamaño de partícula de la EC molida, se llevó a cabo mediante difracción láser (ADL, Mastersizer 2000, Malvern Instruments). La composición química de la EC se obtuvo mediante fluorescencia de rayos-X (FRX, Magic Pro, Philips, mod. PW2400). Las fases cristalinas existentes en la EC se determinaron por difracción de rayos-X (DRX, Brucker AXS D8 Advance), usando radiación $\mathrm{K} \alpha$ de $\mathrm{Cu}$ y filtro de níquel, con voltaje de $40 \mathrm{kV}$ e intensidad de corriente de $20 \mathrm{~mA}$, en el rango $2 \theta=5-70^{\circ}$, con un ángulo de paso de $0,02^{\circ}$ y tiempo de paso de 2 s/paso. La variación de masa de la EC con el aumento de la temperatura se determinó por análisis termogravimétrico (ATG, Mettler-Toledo 850) en crisoles de alúmina en el intervalo de temperaturas de $35{ }^{\circ} \mathrm{C}$ a $1000{ }^{\circ} \mathrm{C}$, con una velocidad de calentamiento de $20^{\circ} \mathrm{C} \cdot \mathrm{min}^{-1}$ en atmósfera de aire. La muestra molida de EC se estudió por espectroscopia infrarroja por transformada de Fourier en el intervalo $4000-400 \mathrm{~cm}^{-1}$ (FTIR, Brucker Tensor 27 MIR, rango de $7800-370 \mathrm{~cm}^{-1}$ ). El estudio morfológico se realizó con un microscopio de barrido de emisión de campo (FESEM, Zeiss Ultra-55). Para la determinación del residuo insoluble (RI) en ácido clorhídrico y en hidróxido de potasio se siguió lo sugerido por UNE-EN 196-2 (2014), y en la determinación de la densidad real de de la EC, se siguió la norma UNE 80103 (2013). La determinación de la pérdida al fuego (PF) se hizo en una mufla (Carbolite, RHF 1500) a $950{ }^{\circ} \mathrm{C}$ durante $1 \mathrm{~h}$.

\section{RESULTADOS Y DISCUSIÓN}

\subsection{Caracterización química}

En la Tabla 2 se muestra la composición química determinadas mediante FRX de las EC estudiadas. Las EC están compuestas predominantemente por $\mathrm{Fe}_{2} \mathrm{O}_{3}$ y $\mathrm{SiO}_{2}$. Las EC-PN, EC-PQ y EC-NT tienen alto contenido de $\mathrm{CaO}$ debido a la mineralogía de la materia prima usada en la fundición y, por otra, al diseño y al nivel de control del proceso metalúrgico. Además, en la Tabla 2 se muestra que las 
TABla 2. Composición química y otros parámetros físicos de las escorias de cobre estudiadas, $\%$ en peso

\begin{tabular}{|c|c|c|c|c|c|}
\hline & & EC-PN & EC-PQ & EC-CA & EC-NT \\
\hline \multirow{24}{*}{$\begin{array}{l}\text { Composición } \\
\text { química, } \%\end{array}$} & $\mathrm{SiO}_{2}$ & 39,14 & 38,33 & 45,02 & 49,25 \\
\hline & $\mathrm{Fe}_{2} \mathrm{O}_{3}$ & 30,48 & 20,40 & 36,62 & 18,14 \\
\hline & $\mathrm{CaO}$ & 13,41 & 26,10 & 7,72 & 14,32 \\
\hline & $\mathrm{Al}_{2} \mathrm{O}_{3}$ & 8,39 & 7,76 & 5,21 & 8,37 \\
\hline & $\mathrm{MgO}$ & 2,09 & 2,14 & 1,53 & 1,05 \\
\hline & $\mathrm{K}_{2} \mathrm{O}$ & 1,5 & 0,78 & 0,54 & 2,21 \\
\hline & $\mathrm{P}_{2} \mathrm{O}_{5}$ & 1,20 & 0,64 & 0,55 & 0,16 \\
\hline & $\mathrm{Na}_{2} \mathrm{O}$ & 1,05 & 0,64 & - & - \\
\hline & $\mathrm{CuO}$ & 0,57 & 0,69 & 1,24 & 0,72 \\
\hline & $\mathrm{SO}_{3}$ & 0,46 & 0,26 & 0,49 & 0,16 \\
\hline & $\mathrm{TiO}_{2}$ & 0,38 & 0,39 & 0,20 & 0,43 \\
\hline & $\mathrm{ZnO}$ & 0,36 & 0,49 & 0,03 & 1,79 \\
\hline & $\mathrm{BaO}$ & 0,27 & 0,04 & 0,08 & 0,79 \\
\hline & $\mathrm{MnO}$ & 0,24 & 1,05 & 0,10 & 1,02 \\
\hline & $\mathrm{Sb}_{2} \mathrm{O}_{3}$ & 0,14 & 0,02 & - & 0,18 \\
\hline & $\mathrm{Co}_{3} \mathrm{O}_{4}$ & 0,09 & - & 0,17 & 0,07 \\
\hline & $\mathrm{Cl}$ & 0,07 & 0,03 & 0,09 & 0,06 \\
\hline & $\mathrm{P}_{\mathrm{b}} \mathrm{O}$ & 0,05 & 0,10 & 0,03 & 0,39 \\
\hline & $\mathrm{As}_{2} \mathrm{O}_{3}$ & 0,04 & 0,02 & 0,21 & 0,79 \\
\hline & $\mathrm{ZrO}_{2}$ & 0,03 & 0,01 & 0,01 & 0,01 \\
\hline & $\mathrm{SrO}$ & 0,03 & 0,03 & 0,01 & 0,03 \\
\hline & $\mathrm{MoO}_{3}$ & 0,02 & 0,11 & - & 0,03 \\
\hline & $\mathrm{Rb}_{2} \mathrm{O}$ & 0,01 & 0,01 & - & 0,01 \\
\hline & Otros & - & - & 0,15 & 0,02 \\
\hline \multirow{4}{*}{$\begin{array}{l}\text { Otros } \\
\text { Parámetros }\end{array}$} & $\mathrm{PF}^{\mathrm{a}}, \%$ & $-2,11$ & $-1,50$ & $-2,51$ & $-3,41$ \\
\hline & $\begin{array}{l}\mathrm{DR}^{\mathrm{b}}, \\
\mathrm{g} \cdot \mathrm{cm}^{-3}\end{array}$ & 3,10 & 2,96 & 3,36 & 2,96 \\
\hline & $\mathrm{RI}^{\mathrm{c}}, \%$ & 11,45 & 0,95 & 19,32 & 17,96 \\
\hline & $\mathrm{IB}^{\mathrm{d}}$ & 0,33 & 0,61 & 0,18 & 0,27 \\
\hline
\end{tabular}

${ }^{\mathrm{a}}$ Pérdida al fuego; ${ }^{\mathrm{b}}$ Densidad relativa; ${ }^{\mathrm{c}}$ Residuo insoluble;

d Índice de basicidad

EC son ácidas, presentando un índice de basicidad $\mathrm{IB}=(\mathrm{CaO}+\mathrm{MgO}) /\left(\mathrm{SiO}_{2}+\mathrm{Al}_{2} \mathrm{O}_{3}\right)<1$. Por otra parte, estas EC, de ser empleadas en construcción, asegurarían la estabilidad de volumen por expansión de las mezclas conglomerantes basadas en cemento Portland debido a los bajos niveles de $\mathrm{MgO}$ observados (Yang et al., 2010a).

\subsection{Caracterización física}

En la Tabla 2 se indican los resultados de la caracterización física que incluye la densidad real (DR), pérdida al fuego (PF) y el residuo insoluble (RI). Las EC poseen una DR promedio de $3,1{\mathrm{~g} . \mathrm{cm}^{-3}}^{-3}$ que aumenta al aumentar el contenido de $\mathrm{Fe}_{2} \mathrm{O}_{3}$. Además, en todas las EC se evidencia una ganancia de masa en el ensayo de PF. Esta ganancia de masa se explicaría por la oxidación de los
TABla 3. Diámetro medio y tiempo de molienda de las escorias

\begin{tabular}{lcccc}
\hline & EC-PN & EC-PQ & EC-CA & EC-NT \\
\hline $\mathrm{D}_{\text {medio, }}, \mu \mathrm{m}$ & 15,33 & 15,12 & 15,03 & 20,82 \\
$\mathrm{t}_{\text {molienda, }}$ min & 40 & 35 & 55 & 50 \\
$\mathrm{D}_{(0,9)} / \mathrm{D}_{(0,1)}$ & 42,5 & 290,5 & 28,2 & 37,3 \\
\hline
\end{tabular}

compuestos ferrosos Fe (II) y de los sulfuros contenidos en la EC, que se han podido transformar en óxidos férricos $\mathrm{Fe}$ (III) y sulfatos al término de la calcinación (Sánchez de Rojas et al., 2008) que se realiza con atmósfera de aire. Por otra parte, el RI que representa la fracción de la EC que no ha entrado en disolución frente al ataque con ácido clorhídrico e hidróxido de potasio durante el ensayo, muestra un valor bajo $(0,95)$ en la EC-PQ en comparación con las otras EC estudiadas, esto nos indicaría una mayor cantidad de sílice reactiva.

En la Tabla 3 se muestra el diámetro medio de las partículas y el tiempo de molienda de las EC. La relación de los percentiles $\mathrm{D}_{(0,9)} / \mathrm{D}_{(0,1)}$ mostrada en la Tabla 3 es $>10$, lo cual nos indica una distribución de partículas muy amplia (Merkus, 2009). Todas las EC estudiadas superan este índice, siendo particularmente elevado en la EC-PQ. En la Fig. 1 se presentan las curvas de distribución de partículas de las EC. En la Fig. 2 se muestra la morfología de las EC observadas por microscopía electrónica de barrido, en general presentan partículas de un aspecto similar con fracturas de forma irregular debido al proceso de molienda. En la Fig. 3 se presentan los espectros infrarrojos (FTIR) de las escorias molidas. Considerando la actividad de cada muestra en función del número de ondas de los espectros FTIR, se pueden identificar 5 zonas. Las zonas 3 - 5 son las que registran la mayor actividad, siendo común a la totalidad de las EC ensayadas. La zona 1 comprende rangos entre $3750-3550 \mathrm{~cm}^{-1}$, la zona 2 entre $3030-2840 \mathrm{~cm}^{-1}$, la zona 3 entre $1100-750 \mathrm{~cm}^{-1}$, la zona 4 entre $750-610 \mathrm{~cm}^{-1}$ y la zona 5 entre $610-400 \mathrm{~cm}^{-1}$. Los picos en torno a los $2300 \mathrm{~cm}^{-1}$ se deben posiblemente a la presencia de $\mathrm{CO}_{2}$ en el momento del ensayo. El detalle de las bandas FTIR y los posibles enlaces son mostrados en la Tabla 4. En la Fig. 4 se muestran las curvas termogravimétricas (TG) y sus derivadas (DTG) para las distintas EC estudiadas. Se puede observar en las curvas TG que todas las EC sufren ganancias de masa en el rango $35-1000{ }^{\circ} \mathrm{C}$, lo cual probablemente se deba a los procesos de oxidación de los compuestos ferrosos Fe (II) y de los sulfuros contenidos en la EC. Este hecho corroboraría los valores de PF obtenidos. Dicha ganancia, puede tener su origen en la oxidación de la fayalita (Marghussian and Maghsoodipoor, 1999). El pico en torno a los 


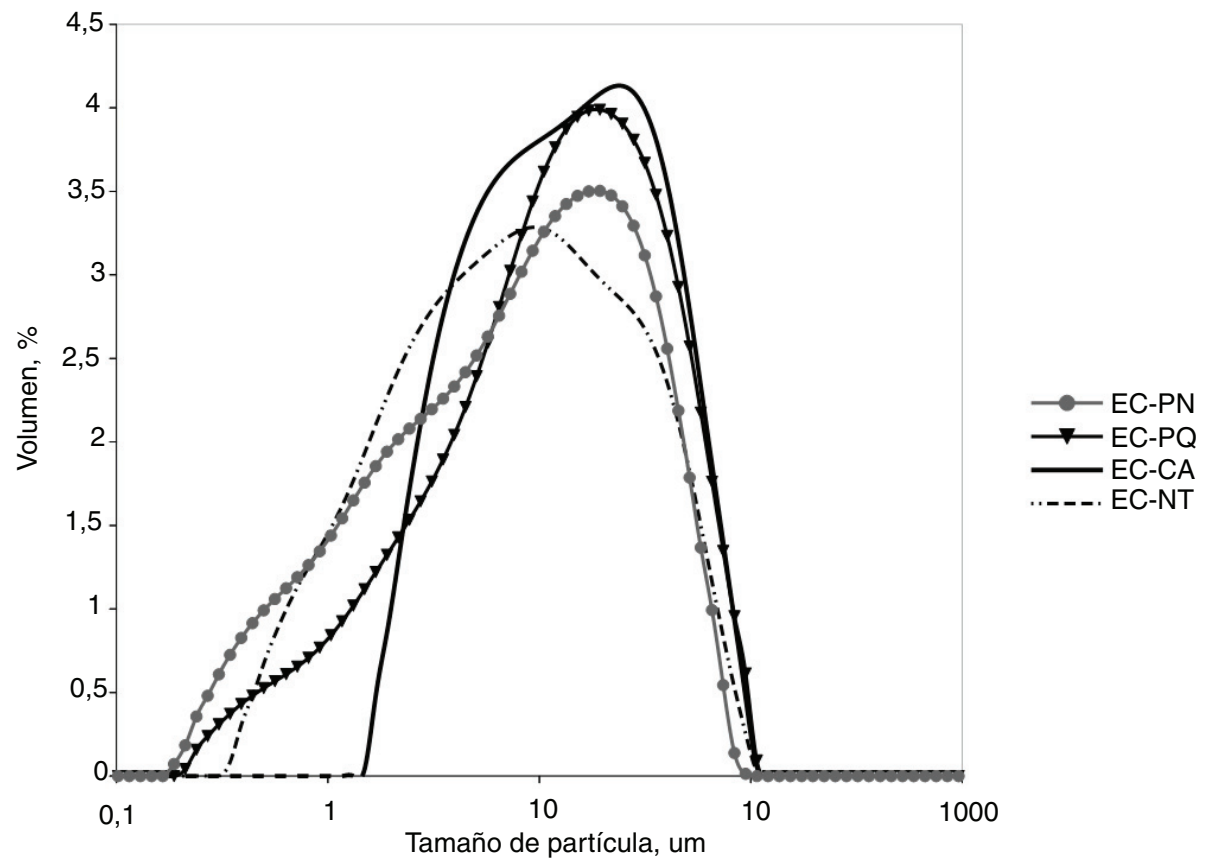

Figura 1. Curvas de distribución de tamaño de partículas de las EC molidas.
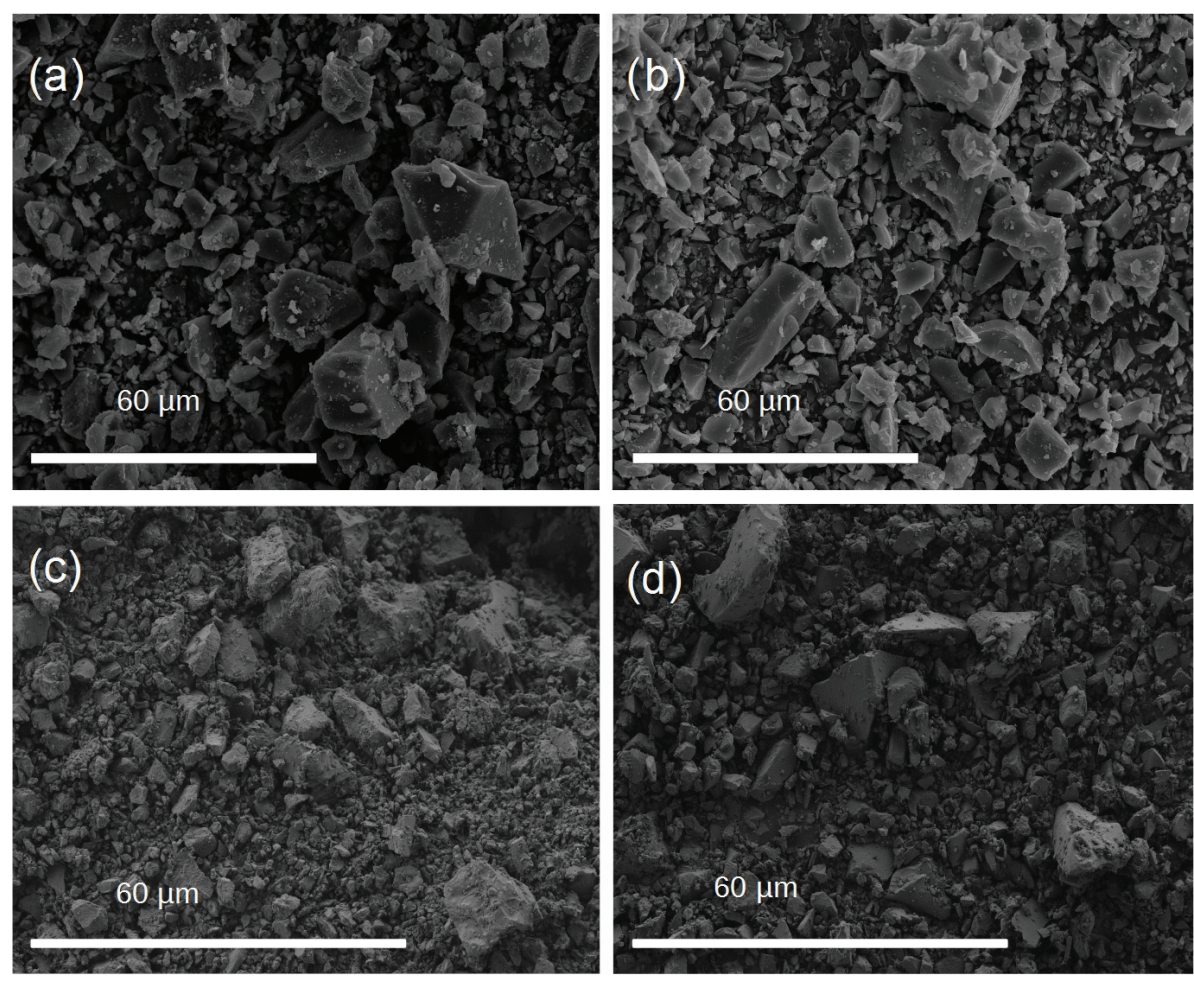

Figura 2. Micrografías a 1000x de las EC molidas: a) EC-PN, b) EC-PQ, c) EC-CA y d) EC-NT.

$500{ }^{\circ} \mathrm{C}$ de la escoria EC-CA puede ser relacionado a las transformaciones de la magnetita a maghemita y los picos en torno a los $760{ }^{\circ} \mathrm{C}$ de EC-PQ y EC-NT, a las transformaciones de maghemita a hematita (Marghussian and Maghsoodipoor, 1999). Las ganancias de masa de las EC obtenidas fueron: 3,15; 2,15; 3,09 y 2,19\% para EC-PN, EC-PQ, EC-CA y EC-NT, respectivamente. 


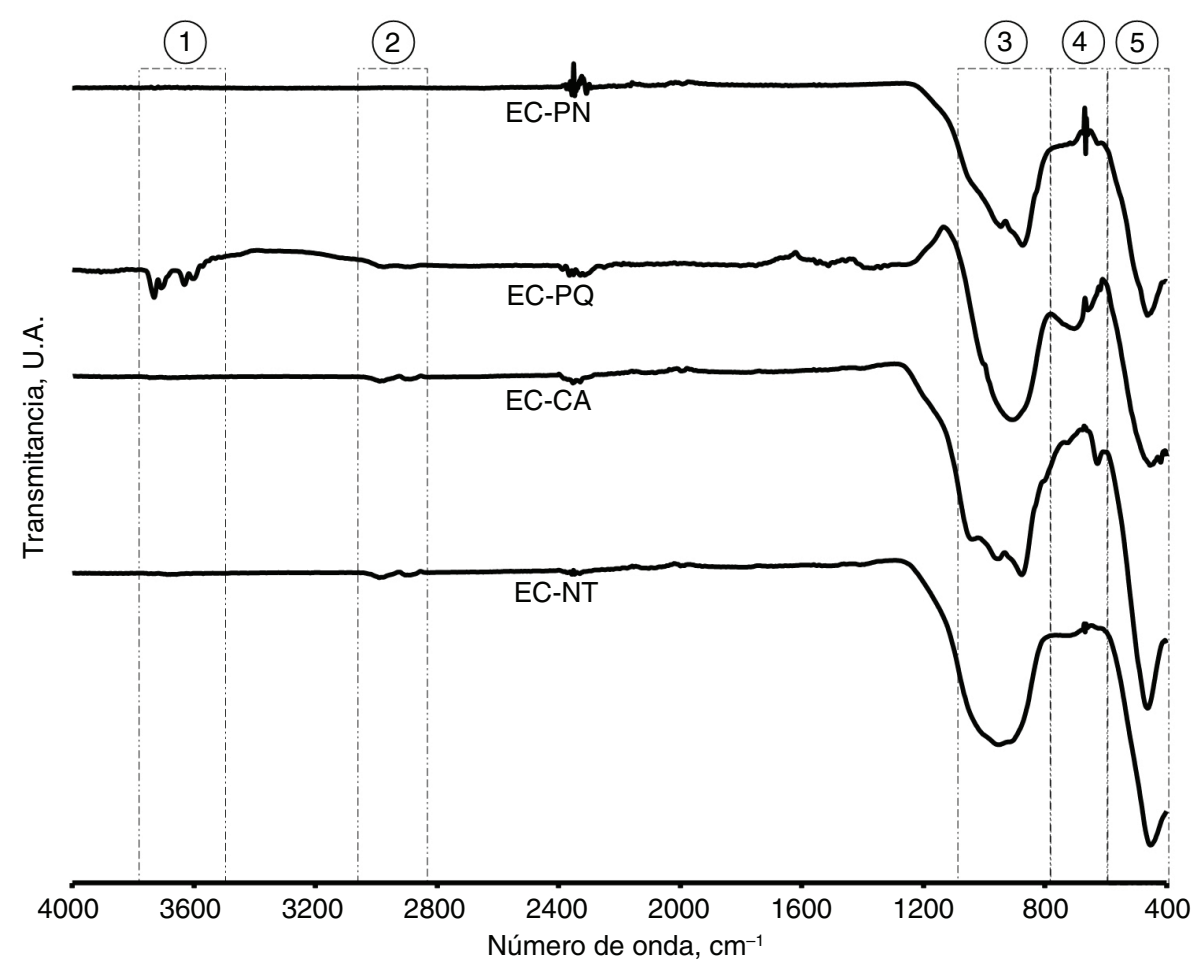

FIGURA 3. Espectros FTIR de las escorias de cobre molidas.

TABLA 4. Bandas FTIR de escorias de cobre

\begin{tabular}{|c|c|c|c|}
\hline Muestra & Número de ondas $\left(\mathrm{cm}^{-1}\right)$ & Vibraciones posibles & Referencia \\
\hline \multirow[t]{3}{*}{ EC-PN } & 1042 & Extensión simétrica y asimétrica de $\mathrm{Si}-\mathrm{O}$ & (Nath y Kumar, 2013) \\
\hline & 950,876 & Extensión asimétrica de $\mathrm{Si}-\mathrm{O}$ del modo $v_{3}$ en $\mathrm{SiO}_{4}$ & (Mihailova y Mehandjiev, 2010) \\
\hline & 463 & $\begin{array}{l}\text { División degenerada de } v_{4} \text { vibraciones de flexión } \\
\text { asimétrica de Si-O }\end{array}$ & (Mihailova y Mehandjiev, 2010) \\
\hline \multirow[t]{8}{*}{ EC-PQ } & $3728-3600$ & Valencia $\mathrm{OH}^{-}$, simétrica y asimétrica & (Darder et al., 2014) \\
\hline & 2972 & -OH del agua & (Sakulich et al., 2009) \\
\hline & 1000,905 & Extensión asimétrica de $\mathrm{Si}-\mathrm{O}$ del modo 03 en $\mathrm{SiO}_{4}$ & (Mihailova y Mehandjiev, 2010) \\
\hline & 704,617 & Enlaces Al-O & (Fernández-Jiménez, 2000) \\
\hline & 659 & Flexión enlaces $\mathrm{Si}-\mathrm{O}-\mathrm{Si}$ & (Fernández-Jiménez, 2000) \\
\hline & 453 & Asociadas a deformaciones $\mathrm{v}_{4}(\mathrm{O}-\mathrm{Si}-\mathrm{O})$ & (Fernández-Jiménez, 2000) \\
\hline & 445 & Flexión de Si-O/Al-O & $\begin{array}{l}\text { (Nath y Kumar, 2013; Komnitsas } \\
\text { et al., 2013) }\end{array}$ \\
\hline & 418 & Enlaces Si-OH & (Velázquez, 2002) \\
\hline \multirow[t]{5}{*}{ EC-CA } & $2988,2975,2900$ & -OH del agua & (Sakulich et al., 2009) \\
\hline & 1041 & Extensión simétrica y asimétrica de $\mathrm{Si}-\mathrm{O}$ & (Nath and Kumar, 2013) \\
\hline & 955,875 & Extensión asimétrica de $\mathrm{Si}-\mathrm{O}$ del modo $v_{3}$ en $\mathrm{SiO}_{4}$ & (Mihailova y Mehandjiev, 2010) \\
\hline & $803,723,625$ & Enlaces Al-O & (Fernández-Jiménez, 2000) \\
\hline & 461 & $\begin{array}{l}\text { División degenerada de } v_{4} \text { vibraciones de flexión } \\
\text { asimétrica de Si-O }\end{array}$ & (Mihailova y Mehandjiev, 2010) \\
\hline \multirow[t]{3}{*}{ EC-NT } & $2990,2975,2900$ & $-\mathrm{OH}$ del agua & (Sakulich et al., 2009) \\
\hline & 951,912 & Extensión asimétrica de $\mathrm{Si}-\mathrm{O}$ del modo $v_{3}$ en $\mathrm{SiO}_{4}$ & (Mihailova y Mehandjiev, 2010) \\
\hline & 453 & Asociadas a deformaciones $v_{4}(\mathrm{O}-\mathrm{Si}-\mathrm{O})$ & (Fernández-Jiménez, 2000) \\
\hline
\end{tabular}



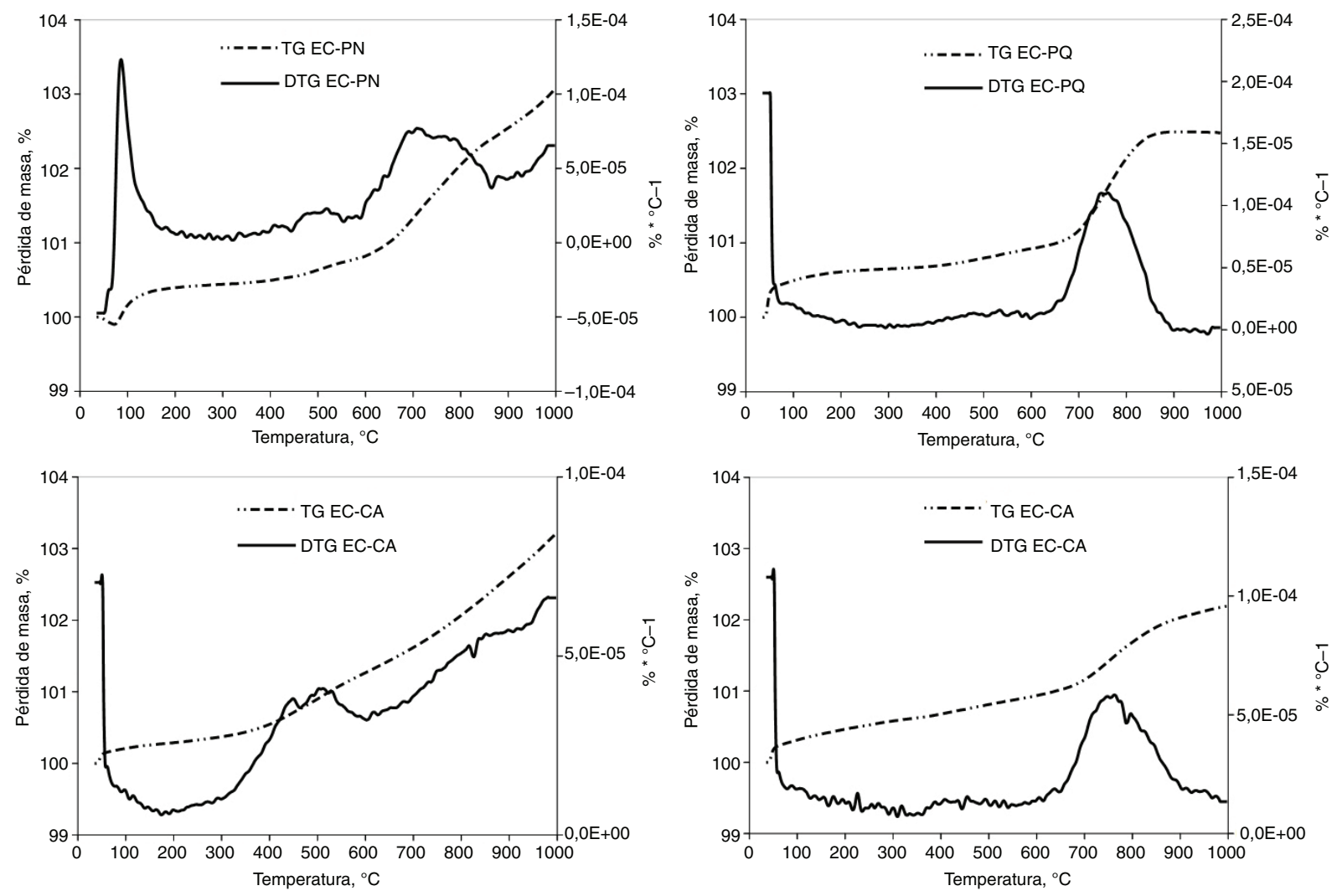

Figura 4. TG y DTG de las escorias de cobre.

\subsection{Caracterización mineralógica}

La Fig. 5 muestra los difractogramas de las EC estudiadas. Los patrones utilizados en la caracterización de estas EC fueron: para el diópsido $\left(\mathrm{CaMgSiO}_{3}\right)$ : PDFcard 190239 , fayalita $\left(\mathrm{Fe}_{2} \mathrm{SiO}_{4}\right)$ : PDFcard 340178, clinoferrosilita $\left(\mathrm{FeSiO}_{3}\right)$ : PDFcard 170548, magnetita $\left(\mathrm{Fe}_{3} \mathrm{O}_{4}\right)$ : PDFcard 190629, cristobalita $\left(\mathrm{SiO}_{2}\right)$ : PDFcard 391425, microclina $\left(\mathrm{KAlSi}_{3} \mathrm{O}_{8}\right.$ ): PDFcard 190926 y wollastonita $\left(\mathrm{CaSiO}_{3}\right)$ : PDFcard 100489. La mineralogía de EC-PN es principalmente una estructura cristalina formada por diópsido, magnetita y fayalita. En menor presencia se encuentra la clinoferrosilita. La EC-PQ es totalmente amorfa, esto se puede verificar por la fuerte desviación en la línea base en el intervalo 20 - 40 grados $2 \theta$. También se detectaron algunas trazas de compuestos cristalinos como diópsido, fayalita y clinoferrosilita. La EC-CA presenta cierto carácter amorfo, verificado por la desviación en la línea base en el intervalo 15 - 25 grados $2 \theta$. Su mineralogía es mayoritariamente fayalita, la cual puede ser observada en el pico de mayor intensidad. Además, están presentes compuestos minoritarios como la magnetita, clinoferrosilita, seguido por la cristobalita. Se detectaron trazas de microclina posiblemente de feldespato. La EC-NT posee una fase amorfa, lo cual puede verificarse por la desviación de la línea base en el rango 15 - 30 grados $2 \theta$. Está constituida principalmente por cristobalita seguida por clinoferrosilita y por compuestos minoritarios como la magnetita, fayalita. Se observaron trazas de wollastonita y trazas de microclina posiblemente de feldespato. La estructura amorfa de una EC nos indica que ésta posee reactividad puzolánica elevada en comparación con una EC de estructura cristalina, por lo tanto, puede ser considerada como un material con propiedades cementantes interesantes por estudiar. La EC-PQ, como se ha visto, es completamente amorfa lo que la hace potencialmente muy reactiva como un cemento puzolánico. Este grado de reactividad se corrobora con el bajo valor obtenido en el ensayo de RI mostrado en la Tabla 2.

\section{CONCLUSIONES}

- Se caracterizaron química, física y mineralógicamente cuatro escorias de cobre de vertederos chilenos abandonados del siglo XIX usando diferentes técnicas de laboratorio. Las EC estudiadas fueron clasificadas químicamente como escorias ácidas de acuerdo al 


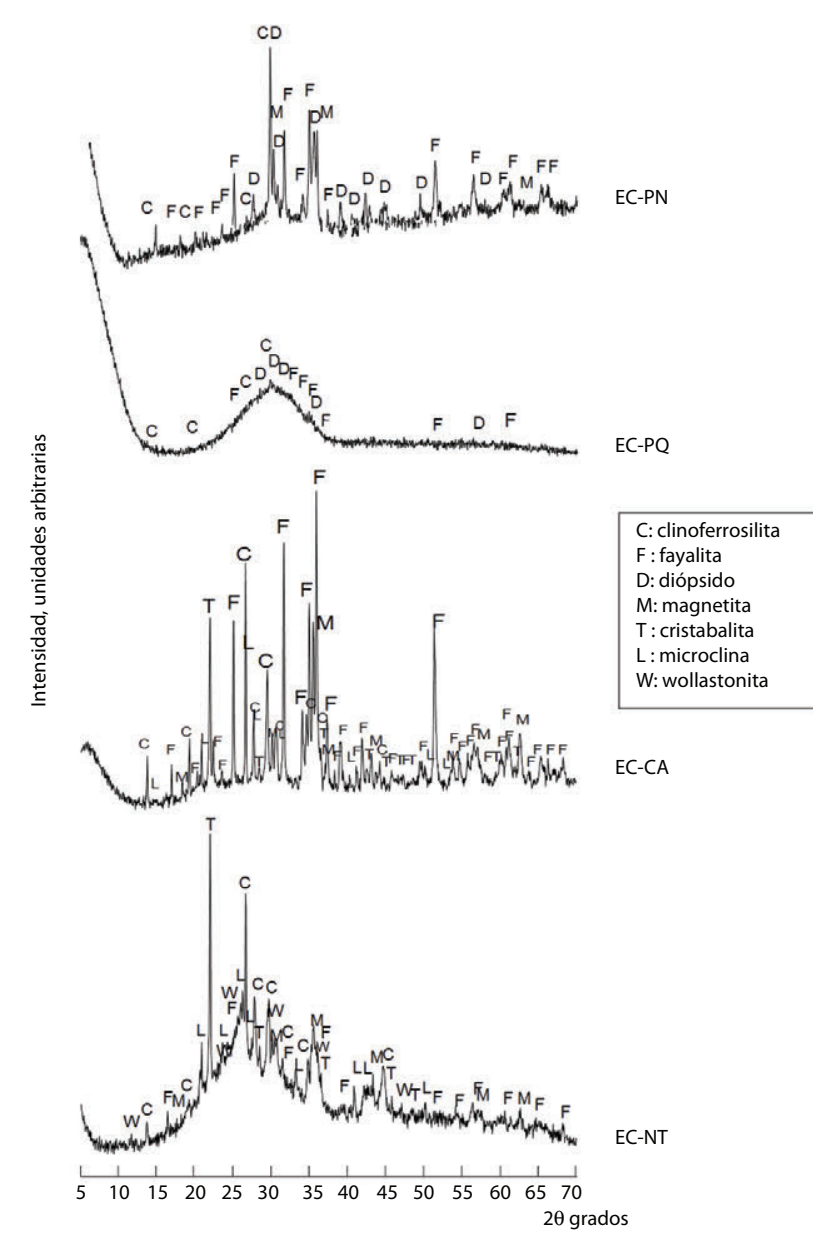

FIgURA 5. Difractogramas de DRX de escorias de cobre.

Índice de Basicidad, con un mayor contenido (\% en peso) de $\mathrm{SiO}_{2}(38-49 \%)$ que de $\mathrm{Fe}_{2} \mathrm{O}_{3}$ (18-37\%) y con una importante cantidad de $\mathrm{CaO}(8-26 \%)$ y $\mathrm{Al}_{2} \mathrm{O}_{3}(5-8 \%)$. Su mineralogía y estructura es variada, la EC-PQ mostró que es amorfa y las EC-PN, EC-CA y EC-NT poseen una estructura principalmente cristalina con cierto carácter amorfo mostrado en la desviación de la línea base en DRX.

- Las fases minerales mayoritarias presentes en las EC correspondieron a diópsido, fayalita, magnetita, cristobalita y clinoferrosilita. Los niveles de calcio indicarían que las escorias podrían poseer propiedades cementantes para ser utilizadas en construcción. Además, el bajo valor de RI obtenido en la EC-PQ junto con su estructura amorfa, nos indicaría una potencial reactividad, por lo que podría ser utilizado en la producción de cementos puzolánicos.

- La gran cantidad de EC disponible en los vertederos del siglo XIX, de aproximadamente 3,5 Mt métricas, así como su apreciable contenido de $\mathrm{CuO}(0,6-1,2 \%)$ haría viable económicamente, también desde el punto de la sostenibilidad, la recuperación de ese metal.

\section{AGRADECIMIENTOS}

A programa de la Unión Europea Erasmus Mundus Lindo por la beca doctoral de D. Amin Nazer y al proyecto DIUDA 22235 por el cofinanciamiento de etapa de molienda de escorias.

\section{REFERENCIAS}

Al-Jabri, K.S., Al-Saidy, A.H., Taha, R. (2011). Effect of copper slag as a fine aggregate on the properties of cement mortars and concrete. Constr. Build. Mater. 25 (2), 933-938. http://dx.doi.org/10.1016/j.conbuildmat.2010.06.090.

Ambler, J.O. (1920). Metalurgical Bookkeeping, Professional Degree, University of Missouri, Rolla, Missouri, USA.

Aracena, F.M. (1884). Apuntes de viaje: La industria del cobre en la Provincias de Atacama y Coquimbo, Valparaíso, Chile.

Ariño, A.M., Mobasher, B. (1999). Effect of ground copper slag on strength and toughness of cementitious mixes. $A C I$ Mater. J. 96 (1), 68-73.

Arslan, C., Arslan, F. (2002). Recovery of copper, cobalt, and zinc from copper smelter and converter slags. Hydrometallurgy 67 (1-3), 1-7. http://dx.doi.org/10.1016/ S0304-386X(02)00139-1.

Banza, A., Gock, E., Kongolo, K. (2002). Base metals recovery from copper smelter slag by oxidising leaching and solvent extraction. Hydrometallurgy 67 (1-3), 63-69. http://dx.doi. org/10.1016/S0304-386X(02)00138-X

Cao, H., Wang, J., Zhang, L., Sui, Z. (2012). Study on Green Enrichment and Separation of Copper and Iron Components from Copper Converter Slag. Procedia Environ. Sci. 16, 740-748. http://dx.doi.org/10.1016/j. proenv.2012.10.101.

Chen, M., Han, Z., Wang, L. (2011). Recovery of valuable metals from copper slag by hydrometallurgy. Adv. Mat. Res. 402, 35-40. http://dx.doi.org/10.4028/www.scientific.net/ AMR.402.35

Choi, S.C., Lee, W.K. (2013). Effect of $\mathrm{CaO}$ and $\mathrm{Fe}_{2} \mathrm{O}_{3}$ on the Geopolymer Made from Mine Tailing and Melting Slag. J. Korea Soc. Waste Manag. 30 (6), 572-577. http://dx.doi. org/10.9786/kswm.2013.30.6.572.

Darder, M., Gonzalez-Alfaro, Y., Aranda, P., Ruiz-Hitzky, E. (2014). Silicate-based multifunctional nanostructured materials with magnetite and Prussian blue: application to cesium uptake. $R S C A d v$. 4, 35415-35421. http://dx.doi. org/10.1039/C4RA06023G.

Fernández-Jiménez, A. (2000). Cementos de escorias activadas alcalinamente: influencia de las variables y modelización del proceso, Tesis Doctoral, Universidad Autónoma de Madrid, Madrid, España.

Jun-wei, S. (2013). Study on the effect of copper slag admixtures to properties and structure of concrete. Inform. Technol. J. 12 (23), 7396-7400. http://dx.doi.org/10.3923/ itj.2013.7396.7400.

Khater, H.M. (2012). Effect of calcium on geopolymerization of aluminosilicate wastes. J. Mater. Civil Eng. 24 (1), 92-101. http://dx.doi.org/10.1061/(ASCE)MT.1943-5533.0000352.

Kim, B.-S., Jo, S.-K., Shin, D., Lee, J.-C., Jeong, S.-B. (2013). A physico-chemical separation process for upgrading iron from waste copper slag. Int. J. Miner. Process. 124, 124-127. http://dx.doi.org/10.1016/j.minpro.2013.05.009.

Komnitsas, K., Zaharaki, D., Bartzas, G. (2013). Effect of sulphate and nitrate anions on heavy metal immobilization in ferronickel slag geopolymers. Appl. Clay Sci. 73, 103-109. http://dx.doi.org/10.1016/j.clay.2012.09.018.

Li, K., Ping, S., Wang, H., Ni, W. (2013). Recovery of iron from copper slag by deep reduction and magnetic beneficiation. Int. J. Miner. Metall. Mater. 20 (11), 1035-1041. http:// dx.doi.org/10.1007/s12613-013-0831-3. 
Marghussian, V., Maghsoodipoor, A. (1999). Fabrication of unglazed floor tiles containing Iranian copper slags. Ceram. Int. 25 (7), 617-622. http://dx.doi.org/10.1016/ S0272-8842(98)00075-3.

Marín Vicuña, S. (1920). La industria del cobre en Chile: Problemas Nacionales, Impr. Universitaria, Santiago de Chile.

Merkus, H. (2009). Particle Size Measurements. Fundamentals, Practice, Quality, Particle Technology Series. Vol 17, Springer, Netherlands.

Mihailova, I., Mehandjiev, D. (2010). Characterization of fayalite from copper slag. J. Univ. Chem. Technol. Metall. 45, 317-326.

Mithun, B.M., Narasimhan, M.C. (2016). Performance of alkali activated slag concrete mixes incorporating copper slag as fine aggregate. J. Clean. Prod. 112 (Part. 1), 837-844. http:// dx.doi.org/10.1016/j.jclepro.2015.06.026.

Murari, K., Siddique, R., Jain, K.K. (2015). Use of waste copper slag, a sustainable material. J. Mater. Cycles Waste 17 (1), 13-26. http://dx.doi.org/10.1007/s10163-014-0254-x.

Nadirov, R.K., Syzdykova, L.I., Zhussupova, A.K., Usserbaev, M.T. (2013). Recovery of value metals from copper smelter slag by ammonium chloride treatment. Int. $J$. Miner. Process. 124, 145-149. http://dx.doi.org/10.1016/j. minpro.2013.07.009.

Nath, S.K., Kumar, S. (2013). Influence of iron making slags on strength and microstructure of fly ash geopolymer. Constr. Build. Mater. 38, 924-930. http://dx.doi.org/10.1016/j. conbuildmat.2012.09.070.

Nazer, A., Fuentes, S., Castillo, P., González, L., Pavez, O., Varela, O., Lanas, O. (2012). Baldosas de escorias de cobre. Innovación en producción limpia. Iberoam. J. Proj. Manag. $3(2), 12$.

Nazer, A., Pavez, O., Toledo, I. (2013). Effect of type cement on the mechanical strength of copper slag mortars. Revista Escola de Minas 66 (1), 85-90. http://www.redalyc.org/articulo.oa?id=56425762011.

Nazer, A., Payá, J., Borrachero, M.V., Monzó, J. (2016). Use of ancient copper slags in Portland cement and alkali activated cement matrices. J. Environ. Manage. 167, 115-123. http://dx.doi.org/10.1016/j.jenvman.2015.11.024

Onuaguluchi, O. (2012). Properties of Cement Based Materials Containing Copper Tailings, Doctoral Dissertation, Eastern Mediterranean University, North Cyprus, Turkey.

Panda, S., Mishra, S., Rao, D.S., Pradhan, N., Mohapatra, U., Angadi, S., Mishra, B.K. (2015). Extraction of copper from copper slag: Mineralogical insights, physical beneficiation and bioleaching studies. Korean J. Chem. Eng. 32 (4), 667-676. http://dx.doi.org/10.1007/s11814-014-0298-6.

Piatak, N.M., Parsons, M.B., Seal II, R.R. (2015). Characteristics and environmental aspects of slag: A review. Appl. Geochem. 57, 236-266. http://dx.doi.org/10.1016/j. apgeochem.2014.04.009.

Ros-Latienda, L., Fernández-Carrasquilla, J. (2013). Caracterización de escorias metalúrgicas procedentes de yacimientos arqueológicos de Navarra (Siglos II a. C.- IV d. C). Rev. Metal. 49 (6), 438-448. http://dx.doi.org/10.3989/ revmetalm.1302.
Rudnik, E., Burzyńska, L., Gumowska, W. (2009). Hydrometallurgical recovery of copper and cobalt from reductionroasted copper converter slag. Miner. Eng. 22 (1), 88-95. http://dx.doi.org/10.1016/j.mineng.2008.04.016.

Sakulich, A.R., Anderson, E., Schauer, C., Barsoum, M.W. (2009). Mechanical and microstructural characterization of an alkali-activated slag/limestone fine aggregate concrete. Constr. Build. Mater. 23, 2951-2957. http://dx.doi. org/10.1016/j.conbuildmat.2009.02.022.

Sánchez de Rojas, M., Rivera, J., Frías, M., Marín, F. (2008). Use of recycled copper slag for blended cements. J. Chem. Technol. Biot. 83 (3), 209-217. http://dx.doi.org/10.1002/ jctb. 1830.

Shi, C., Meyer, C., Behnood, A. (2008). Utilization of copper slag in cement and concrete. Resour. Conserv. Recy. 52 (10), 1115-1120. http://dx.doi.org/10.1016/j. resconrec.2008.06.008.

Shi, C., Qian, J. (2000). High performance cementing materials from industrial slags - A review. Resour. Conserv. Recy. 29 (3), 195-207. http://dx.doi.org/10.1016/ S0921-3449(99)00060-9.

Sociedad Nacional de Minería (1894). Datos estadísticos sobre las minas i fábricas metalúrgicas de la República de Chile correspondientes al año 1893. Publicaciones de la Exposición de Minería i Metalurgia de Santiago, Imprenta Nacional, Santiago de Chile.

Taha, R.A., Alnuaimi, A.S., Al-Jabri, K.S., Al-Harthy, A.S. (2007). Evaluation of controlled low strength materials containing industrial by-products. Build. Environ. 42 (9), 3366-3372. http://dx.doi.org/10.1016/j.buildenv.2006.07.028.

Thomas, B.S., Gupta, R.C. (2013). Mechanical properties and durability characteristics of concrete containing solid waste materials. J. Clean. Prod. 1-6, http://dx.doi.org/10.1016/j. jclepro.2013.11.019.

Tixier, R., Devaguptapu, R., Mobasher, B. (1997). The effect of copper slag on the hydration and mechanical properties of cementitious mixtures. Cement. Concrete Res. 27 (10), 1569 1580. http://dx.doi.org/10.1016/S0008-8846(97)00166-X

UNE 80103 (2013). Test methods of cements. Physical analysis. Actual density determination, AENOR.

UNE-EN 196-2 (2014). Métodos de ensayo de cementos. Parte 2: Análisis químico de Cementos, AENOR.

Velázquez, S. (2002). Aplicaciones del catalizador de craqueo catalítico usado (FCC) en la preparación de conglomerantes hidráulicos. Estudio de sus propiedades puzolánicas. Tesis Doctoral, Universitat Politècnica de València, España.

Vicuña Mackenna, B. (1882). El libro de la plata. Imprenta Cervantes, Santiago de Chile.

Yang, H., Fang, K., Tu, S. (2010a). Copper slag with high MgO as pozzolanic material: Soundness, pozzolanic activity and microstructure development. J. Wuhan Univ. Technol. 32 (17), 94-98.

Yang, Z., Rui-lin, M., Wang-dong, N., Hui, W. (2010b). Selective leaching of base metals from copper smelter slag. Hydrometallurgy 103 (1-4), 25-29. http://dx.doi.org/10.1016/j. hydromet.2010.02.009. 